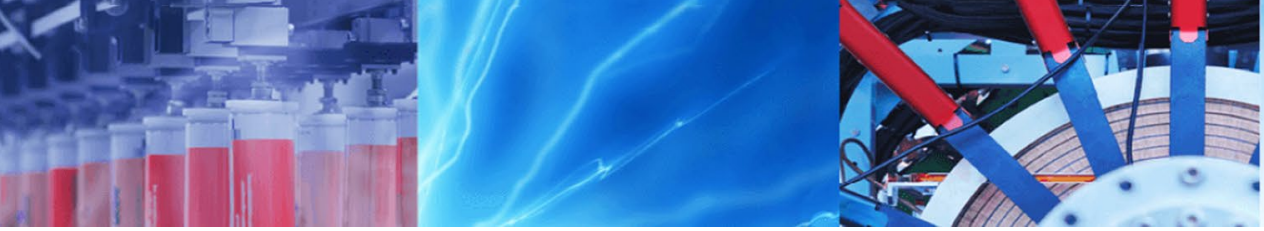

Research Article

\title{
ZnTe/ZnSe heterostructures: In-situ synthesis, characterization and photocatalytic activity for Congo Red degradation
}

\author{
Muhammad Fahad Ehsan ${ }^{1}$ (D) Samia Qudoos ${ }^{1} \cdot$ Zahoor Ahmad $^{4} \cdot$ Saher Hamid $^{2} \cdot$ Muhammad Arfan $^{1} \cdot$ Adeel Zia $^{1}$. \\ Khaula Umbreen ${ }^{3} \cdot{\text { Muhammad Naeem } \text { Ashiq }^{3} \text {. Deependra Tyagi }}^{2}$
}

(c) Springer Nature Switzerland AG 2019

\begin{abstract}
Recently, there is growing concern over environmental pollution rising specifically from the water contamination due to industrial wastes that primarily comprise of organic dyes such as the Congo Red (CR) dye. On the other hand, semiconductors based nanomaterials have proven to be a powerful remediation tool for the polluted environment. Here in, we report an in situ approach for the development of strong interface via one pot hydrothermal synthesis of ZnTe/ZnSe heterostructures by precisely adjusting the chalcogens ( $\mathrm{Te} / \mathrm{Se}$ ) concentration. The as-synthesized photocatalysts have further been characterized via various analytical techniques including X-ray diffraction for the phase confirmation, scanning electron microscopy together with energy dispersive $X$-ray spectroscopy for the morphological as well compositional analysis, and X-ray photoelectron spectroscopy along with UV-Vis diffuse reflectance spectroscopy to plot the alignment of energy levels. Finally, the synthesized heterostructures have been employed as potential photocatalysts for the UV-visible induced degradation of the $C R$ dye. The results reveal remarkable efficacy where heterostructured photocatalysts exhibit enhanced degradation efficiency for up to $\sim 94 \%$ in $65 \mathrm{~min}$. The synergy between the individual counterparts in heterostructure i.e. ZnTe and ZnSe, via the formation of a strong interface plays a significant role in photocatalytic CR fixation by suppressing the charge recombination which is also the key issue in these semiconductor nanomaterials.
\end{abstract}

Keywords Nanomaterials $\cdot$ Photocatalysis $\cdot \mathrm{ZnTe} \cdot \mathrm{ZnSe} \cdot$ Heterostructure

\section{Introduction}

The textile industries are considered as the primary source of release of dyes and effluents in the ecosystem. Among various classes of dyes, the most used dyes especially in the textile industry are azo dyes and hence they are considered to be the most detrimental class of organic pollutants due to their hazardous nature. In the past few decades, the wastewater effluents from dyes and textile industries pose major environmental concern mainly due to their strong color, the existence of high levels of aromatic rings as well as the existence of dissolved solids which makes them potentially more harmful and toxic [1]. $\mathrm{CR}$ is one of the most important azo dyes being extensively used in textile industries for dyeing of fabrics and paper, and also is several other dyeing industries. CR is highly soluble in water and show high persistence in the environment. Once released, it is readily absorbed by the

Electronic supplementary material The online version of this article (https://doi.org/10.1007/s42452-019-0220-2) contains supplementary material, which is available to authorized users.

$\triangle$ Muhammad Fahad Ehsan, m.fahad.ehsan@gmail.com; m.fahad.ehsan@sns.nust.edu.pk; $\bowtie$ Muhammad Naeem Ashiq, naeembzu@bzu.edu.pk | 1 School of Natural Sciences (SNS), National University of Sciences and Technology (NUST), Islamabad 44000, Pakistan. ${ }^{2}$ Verschuren Centre for Sustainability in Energy and the Environment, Cape Breton University, 1250 Grand Lake Road, Sydney, NS B1P 6L2, Canada. ${ }^{3}$ Institute of Chemical Sciences (ICS), Bahauddin Zakariya University (BZU), Multan 60800, Pakistan. ${ }^{4}$ Department of Chemistry, University of Engineering and Technology (UET), Lahore 54890, Pakistan.

SN Applied Sciences (2019) 1:197| https://doi.org/10.1007/s42452-019-0220-2

Received: 4 December 2018 / Accepted: 25 January 2019 / Published online: 1 February 2019 
skin. Furthermore, these dyes also possess high resistance to wastewater treatment techniques such as bacterial and fungal biosorption and bio-degradation in aerobic or anaerobic environment [2, 3]. Several physical, biological and chemical methods have been carried out during the past few decades for their treatment. However, transformation of contaminants from one phase into the other is one of the main disadvantages of using these conventional methods [3]. In the recent past, advanced oxidation processes (AOP) have gathered much attention and are regarded as an alternative treatment strategy for the complete photo-degradation of azo dyes [4, 5].

While using the semiconductor nanomaterials, photocatalysis is considered as a green approach for the environmental remediation and the solar energy conversion i.e. simultaneous pollutant degradation and hydrogen production, mainly to cope with the energy and environmental crisis [6]. Therefore, many efforts are carried out on the development of photocatalysts which can be efficiently utilized for the degradation of pollutants. So far, various photocatalytic materials under different experimental conditions have been utilized for the organic pollutant degradation. Nevertheless, anatase $\mathrm{TiO}_{2}$ has been classified as the most promising material for hydrogen production and degradation of many pollutants [7]. In contrast to this, $\mathrm{TiO}_{2}$ has certain limitations owing to its larger band gap that makes it UV-responsive which constitutes to only $\sim 4 \%$ of the electromagnetic spectrum [8]. Besides, several other oxides of zinc, cadmium, cerium, vanadium, chromium and tin have also been widely used for organic pollutant fixation because of their capacity to absorb wide range of wavelength of solar spectrum [9]. Several researchers across the globe have previously reported a variety of photocatalysts including simple oxides $\mathrm{Bi}_{2} \mathrm{WO}_{6}[10]$ and $\mathrm{WO}_{3}$ [11], complex oxides like $\mathrm{Bi}_{2} \mathrm{WO}_{6}$ [12], sulphides CdS [13] and Nitrides $C_{3} \mathrm{~N}_{4}$ [14] for enhanced solar energy utilization to investigate their photocatalytic activity. In addition, many efforts have also been made so far on designing the visible-light active photocatalysts but there are certain disadvantages such as short life span of photogenerated electrons-hole pairs which in turn limit their practical realization [13]. The recombination of photo-generated electron-hole pairs during photocatalysis is considered as one of the most important factors that mainly govern photocatalytic activity. The recombination of the photo generated charge carriers must be controlled for enhancing the photocatalytic activity [15].

Several strategies are employed in the past to minimize the electron-hole recombination. However, the heterostructure formation has gained the attention of the researchers which effectively improves the activity of photocatalyst by promoting the effective charge separation [16-19]. Many composites such as carbon based materials [20], $\mathrm{AgBr}-\mathrm{Ag}-\mathrm{Bi}_{2} \mathrm{WO}_{6}[21,22]$ and $\mathrm{Bi}_{2} \mathrm{WO}_{6}$ superstructures decorated with $\mathrm{Bi}_{2} \mathrm{O}_{3}$ nanoparticles [23] have been synthesized for photocatalytic applications. Furthermore, several metal oxides based hybrid materials such as $\mathrm{CuO}-\mathrm{TiO}_{2}$ [24], $\mathrm{WO}_{3}-\mathrm{TiO}_{2}$ [25], $\mathrm{ZnO} / \mathrm{TiO}_{2}$ [26], $\mathrm{ZnO} / \mathrm{SnO}_{2}$ [16] and $\mathrm{TiO}_{2} / \mathrm{MgO}$ [27] were also synthesized as potential photocatalysts.

On the other hand, semiconductors of type II-VI ( $\mathrm{Zn}, \mathrm{Cd}$, $\mathrm{Hg}$; and $\mathrm{S}, \mathrm{Se}, \mathrm{Te}$ ) have been proven to be the most promising class of chalcogenide materials for the solar energy conversion applications as well as are viable constituent material in several electronic devices [28-31]. Among others, ZnSe with the bandgap of $\sim 2.7 \mathrm{eV}$ has been extensively utilized for photocatalytic dye degradation and for water splitting [32].

In the present work, for the first time to the best of our knowledge, we have used an in situ hydrothermal approach to combine ZnSe with zinc telluride (ZnTe). ZnTe is a p-type semiconductor having a direct band gap value of $2.20 \mathrm{eV}$ at $300 \mathrm{~K}$ and is a promising material owing to its wide range of applications in solar cells [33], blue-green Led's [34], and in optoelectronic devices [35]. ZnTe is also considered as an attractive material in photocatalysis for its applications in $\mathrm{CO}_{2}$ photoreduction [36-39].

\section{Experimental}

\subsection{Chemicals and reagents}

Zinc powder (99.8\%), tellurium powder (99.8\%), selenium powder (99.6\%), potassium hydroxide (analytical grade, $>85 \%$ ) and absolute ethanol (>99.5\%) were used for the synthesis of materials. These chemicals and reagents were purchased from Sigma Aldrich and were used as such without any further purification. Furthermore, deionized water was used during all the synthesis.

\subsection{Synthesis of photocatalysts}

In total, 5 different catalysts were synthesized including two bare ZnTe and ZnSe (further labelled as ZT and ZS, respectively) along with three $Z n T e / Z n S e$ hybrids (further labelled as ZTS 1, ZTS 2 and ZTS 3) with details given in Table 1. In a typical procedure, $0.1 \mathrm{M}$ zinc powder was dispersed to a $3 \mathrm{M} \mathrm{KOH}$ solution followed by the addition of different concentrations of the tellurium and selenium powders as given in the Table 1. The resulting mixture was then transferred to Teflon-lined tubes filling $80 \%$ of their capacity. These tubes were sealed in the stainless steel high-pressure autoclaves which were further heated in oven at $120^{\circ} \mathrm{C}$ for $2 \mathrm{~h}$. The obtained precipitates were centrifuged, washed several times with deionized water and 
Table 1 Details of precursor concentrations for the synthesis of photocatalysts

\begin{tabular}{llll}
\hline Sample ID & Zn conc. (M) & Te conc. (M) & Se conc. (M) \\
\hline ZT & 0.1 & 0.1 & 0.0 \\
ZTS 1 & 0.1 & 0.075 & 0.025 \\
ZTS 2 & 0.1 & 0.05 & 0.05 \\
ZTS 3 & 0.1 & 0.025 & 0.075 \\
ZS & 0.1 & 0.0 & 0.1 \\
\hline
\end{tabular}

finally with absolute ethanol. The obtained precipitates were dried in vacuum oven at $70^{\circ} \mathrm{C}$. The dried powders were finally ground and stored for further characterizations and photocatalytic applications.

The reaction steps involved during the synthesis of ZnTe/ZnSe have also been proposed in Eqs. 1-6. It can be seen that when zinc powder chemically interacts with $\mathrm{KOH}$ at high temperature and pressure to form potassium zincate with the liberation of hydrogen gas (Eq. 1). This zincate is then dissociated into the constituent ions which are mobilized in the reaction system (Eq. 2). In addition, the respective tellurium and selenium powders, at elevated reaction conditions, react with $\mathrm{KOH}$ to give respective potassium tellurite/selenite which are water soluble and thus are dissociated into the anion species of their respective oxides (Eqs. 3-4) which are further reduced to give $\mathrm{Te}^{2-} / \mathrm{Se}^{2-}$ (Eq. 5). Finally, these anions react together with $\mathrm{Zn}^{2+}$ to give the composite materials as final catalysts.

$$
\begin{aligned}
& \mathrm{Zn}+\mathrm{KOH}+\mathrm{H}_{2} \mathrm{O} \stackrel{\Delta}{\longrightarrow} \mathrm{K}_{2} \mathrm{Zn}(\mathrm{OH})_{4}+\mathrm{H}_{2} \\
& \mathrm{~K}_{2} \mathrm{Zn}(\mathrm{OH})_{4} \stackrel{\Delta}{\longrightarrow} 2 \mathrm{~K}^{+}+\mathrm{Zn}^{2+}+4 \mathrm{OH}^{-} \\
& \mathrm{Te} / \mathrm{Se}+\mathrm{KOH} \stackrel{\Delta}{\longrightarrow} \mathrm{K}_{2} \mathrm{Te} / \mathrm{K}_{2} \mathrm{Te}+\mathrm{K}_{2} \mathrm{TeO}_{3} / \mathrm{K}_{2} \mathrm{SeO}_{3}+\mathrm{H}_{2} \mathrm{O} \\
& \mathrm{K}_{2} \mathrm{TeO}_{3} / \mathrm{K}_{2} \mathrm{SeO}_{3} \stackrel{\mathrm{H}_{2} \mathrm{O}}{\longrightarrow} 2 \mathrm{~K}^{+}+\left[\mathrm{TeO}_{3}\right]^{2-} /\left[\mathrm{SeO}_{3}\right]^{2-} \\
& {\left[\mathrm{TeO}_{3}\right]^{2-} /\left[\mathrm{SeO}_{3}\right]^{2-}+\mathrm{H}_{2} \stackrel{\Delta}{\longrightarrow} \mathrm{Te}^{2-} / \mathrm{Se}^{2-}+\mathrm{O}_{2}+\mathrm{H}_{2} \mathrm{O}} \\
& \mathrm{Zn}^{2+}+\mathrm{Te}^{2-} / \mathrm{Se}^{2-} \stackrel{\Delta}{\longrightarrow} \mathrm{ZnTe} / \mathrm{ZnSe}
\end{aligned}
$$

\subsection{Characterization}

The crystal structure of the synthesized nanomaterials has been probed using X-ray diffraction (XRD) while using Bruker $\mathrm{D}-8$ diffractometer with $\mathrm{Ni}$-filtered $\mathrm{Cu}$-Ka radiation. The diffraction patterns were collected in the $2 \theta$ range of $20^{\circ}-80^{\circ}$ at a scanning rate of $0.1 \% \mathrm{~min}$. Lambda $750 \mathrm{UV} /$ visible/NIR spectrophotometer with $\mathrm{BaSO}_{4}$ as the reference was used to investigate the optical properties in the wavelength ranging from $250-800 \mathrm{~nm}$. For the morphological analysis, Hitachi S4800 field-emission scanning electron microscope (FESEM) was used along with energy dispersive $X$-ray spectrometer (EDX) as well as X-ray photoelectron spectroscopy (XPS) using ESCALAB 250Xi X-ray photoelectron spectrophotometer to study the composition of the as-synthesized materials. In addition, XPS was also performed to determine the valance band positions of the catalysts.

\subsection{Photocatalytic activity}

The as-synthesized ZnTe/ZnSe heterostructures were employed as potential photocatalysts against the degradation of CR dye under UV-Vis solar illumination. The UV-Vis lamp was used as a source of light for carrying out degradation activity. The Lamp was placed $15 \mathrm{~cm}$ above the beaker of $100 \mathrm{~mL}$ capacity with $10 \mathrm{mg}$ of photocatalyst (ZT, ZS, ZST 1 , ZST 2 and ZST 3) dispersed in $80 \mathrm{~mL}$ of 100 ppm CR. In order to establish equilibrium, the catalyst-dye suspension was stirred for about $10 \mathrm{~min}$ in the dark. The solutions were then exposed to UV-visible light irradiations for $65 \mathrm{~min} .5 \mathrm{~mL}$ of the suspension was taken out after every 5 min interval, centrifuged and analyzed with respect to $C R$ concentration as a function of time using UV-Vis spectrophotometer.

\subsection{Calculations}

CR degradation efficiency (\%) was determined while using the following equation:

$\%$ Degradation $=\frac{\text { initial absorbance }- \text { final absorbance }}{\text { initial absorbance }} \times 100$

In addition, different crystalline parameters i.e. crystallite size $(D)$, lattice constants and cell volume $(V)$ of ZnTe and $\mathrm{ZnSe}$, have been calculated by using XRD data. Crystallite size was calculated with the help of Scherrer equation (Eq. 8) while the lattice constant and cell volume were calculated by using Eqs. (9-10). Furthermore, Kubelka-Munk function (Eq. 11) was also used to determine the bandgap energy (eV).

$D=\frac{K \lambda}{\beta \cos \theta}$

$\frac{1}{d^{2}}=\frac{h^{2}+k^{2}+l^{2}}{a^{2}}$

$V=a^{3}$

$\alpha h v^{1 / 2}=K\left(h \nu-E_{g}\right)$ 
Here $K$ is a constant, $\lambda$ is the wavelength for the $X$-ray radiation $(0.15418 \mathrm{~nm}$ for $\mathrm{Cu}-\mathrm{Ka}), \beta$ is the full width at half maxima (FWHM) for the diffraction peak measured at $2 \theta$, and $\theta$ is the diffraction angle. In addition, In addition, $h$ is the Plank's constant, $v$ is the vibrational frequency, $a$ is the absorption coefficient and $E_{g}$ is the bandgap energy. In order to calculate the lattice constant (a) and cell volume $(\mathrm{V})$ for hexagonal $\mathrm{ZnSe}$, following equations were employed.

$\frac{1}{d^{2}}=\frac{4}{3}\left(\frac{h^{2}+h k+k^{2}}{a^{2}}\right)+\frac{p^{2}}{c^{2}}$

$V=0.866 a^{2} c$

\section{Results and discussion}

The crystal structure of the as-synthesized photocatalysts has been explored by XRD crystallography and the results are given in Fig. 1. For ZT (Fig. 1a), the XRD pattern reveals a perfect match with the available diffraction pattern (JCPDS file no. 15-0746). Furthermore, there were no impurity peaks for elemental $\mathrm{Zn}$ or Te to further confirm the presence of single cubic phase. For ZS (Fig. 1e), the results also reveal that all the peaks are well-matched with the standard patterns (JCPDS \# 00-89-2940) corresponding to the hexagonal $\mathrm{ZnSe}$. The sharp and narrow peaks for both ZnTe and ZnSe demonstrate the high crystallinity of the synthesized materials. In addition, the XRD patterns for the heterostructures (ZTS 1-ZTS 3) are also presented in Fig. $1 \mathrm{~b}-\mathrm{d}$. It can clearly be observed from these patterns that all the peaks are contributed either from the

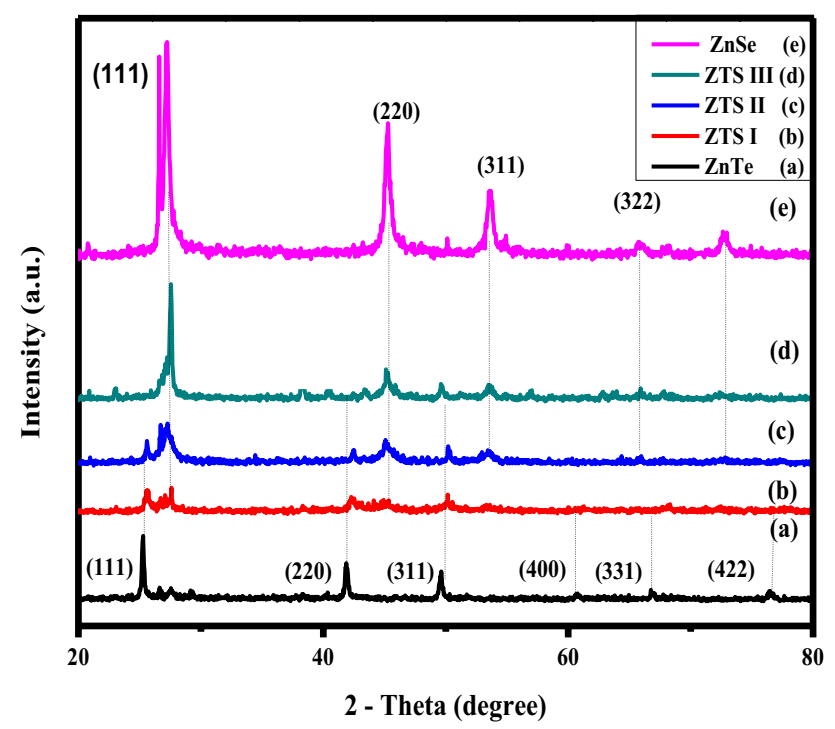

Fig. 1 XRD patterns of a ZT, b ZTS 1, c ZTS 2, d ZTS 3, e ZS cubic ZT or from the hexagonal ZS. No additional peaks are present in the composite materials thereby confirming their phase purity and successful one-pot synthesis only by adjusting the precursor concentrations. It can also be seen from these patterns (Fig. 1b-d) that the intensity of (111) peak corresponding to ZS in Fig. 1e keeps on increasing with a decrease in the ZT (111) peak thereby giving rise to $\mathrm{ZnSe}$ formation in the composites. These XRD patterns (Fig. 1a, e) have also been used for the detailed analysis of various structure parameters and the results are presented in Table 2.

Scanning electron microscopy (SEM) analysis was carried out to investigate the morphology of the synthesized materials and the results are given in Fig. 2. It was observed that ZnTe (Fig. 2a) has irregular morphology comprising of some distorted spheres and nanoparticles. On the other hand, it can be observed in Fig. 2e that ZnSe shows the flakes-like structures. However, both these morphologies are visible in Fig. $2 b-d$ with the increasing density of ZnSe flakes with increasing ZnSe content in the heterostructures.

Energy dispersive X-ray spectroscopy (EDX) also helped to determine the composition and elemental distribution of all the synthesized materials and the results are shown in Fig. S1 in the supplementary information. No extra peaks other than $\mathrm{Zn}$ and Te can be seen in Fig. S1a thereby confirming its impurity free synthesis. This is also observed for the ZnSe in Fig. S1e showing Zn and Se as the main constituent peaks. However, both Te and Se peaks are present in the EDX spectra [Fig. $\mathrm{S} 1(\mathrm{~b}-\mathrm{d})$ ] attributed to the formation of the heterostructures. Moreover, no extra peak is observed which indicate that all samples are highly pure except two small peaks of carbon and silicon which are ascribed to come from sampling preparing procedure.

In addition to the EDX spectroscopy, composition of the as-synthesized materials with respect to the oxidation states of the constituent elements was also confirmed via the XPS analysis and the results are presented in Fig. 3. As evident from the Fig. 3a, Zn, in ZnTe, shows two characteristic peaks at 1044.8 and $1021.26 \mathrm{eV}$ corresponding to $2 \mathrm{P}_{5 / 2}$ and $2 \mathrm{P}_{3 / 2}$ shell, respectively for its $2+$ oxidation state. For Te, its $3 d$ shell XPS scan shows four peaks as shown in Fig. 3b. However, only two peaks at 582.76 and $572.46 \mathrm{eV}$ are responsible for its 2- oxidation state in ZnTe whereas

Table 2 Crystalline parameters derived from XRD patterns for ZnTe and $\mathrm{ZnSe}$

\begin{tabular}{llll}
\hline Sample & $\begin{array}{l}\text { Crystallite size (D) } \\
(\mathrm{nm})\end{array}$ & $\begin{array}{l}\text { Lattice constant } \\
(\mathrm{a})(\AA)\end{array}$ & $\begin{array}{l}\text { Cell vol- } \\
\text { ume }\left(\mathrm{a}^{3}\right) \\
\left(\AA^{3}\right)\end{array}$ \\
\hline ZT & 53.5 & 6.09 & 226.76 \\
ZS & 64.4 & 5.67 & 182.28 \\
\hline
\end{tabular}




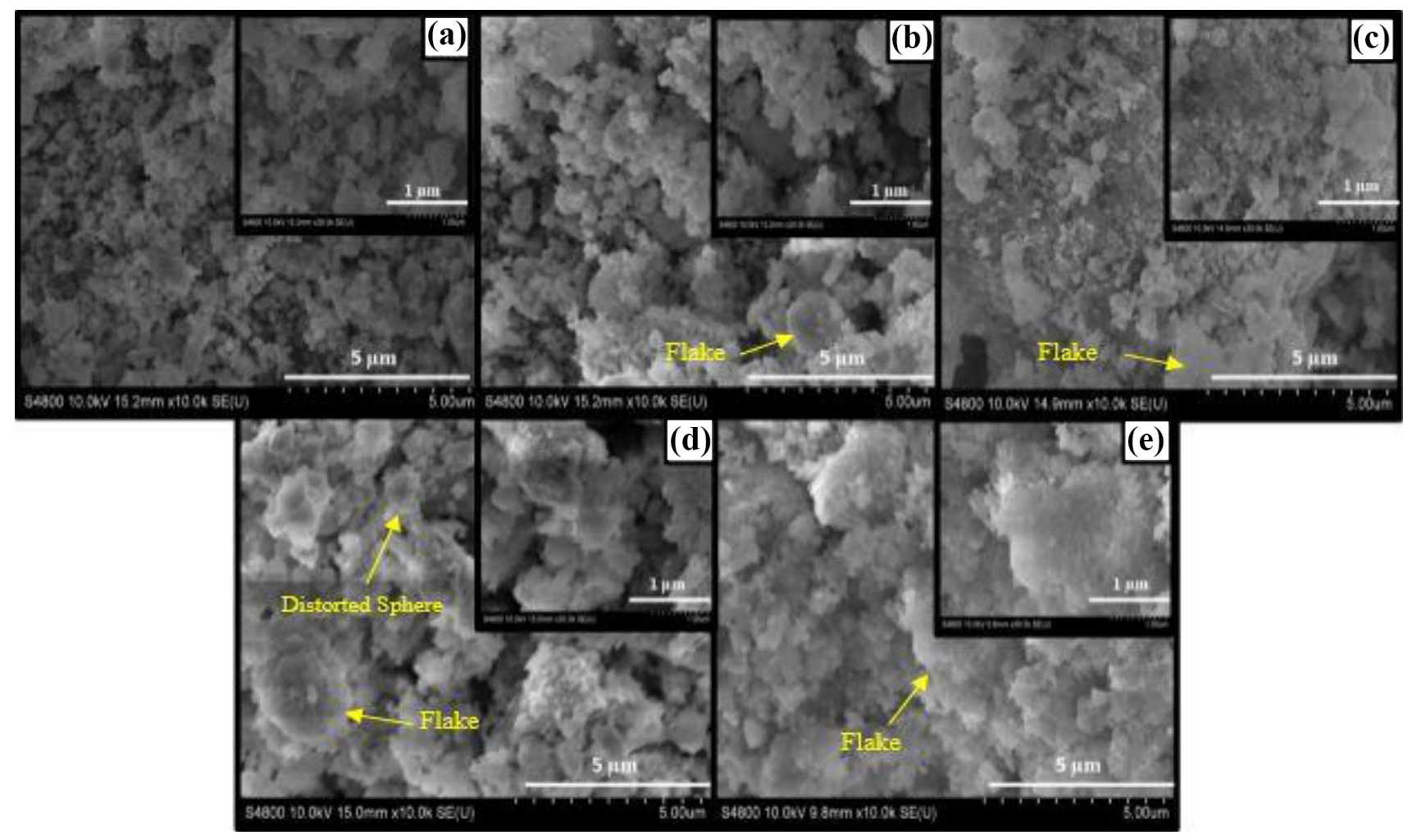

Fig. 2 SEM micrographs of a ZT, b ZTS 1, c ZTS 2, d ZTS 3, e ZS with high magnification images in the insets

Fig. 3 XPS Spectra of the respective elements in assyntehsized photocatalysts: a $\mathrm{Zn} 2 p$ in ZnTe, b Te $3 d, \mathbf{c}$ Zn $2 p$ in $\mathrm{ZnSe}$, d Se $3 d$
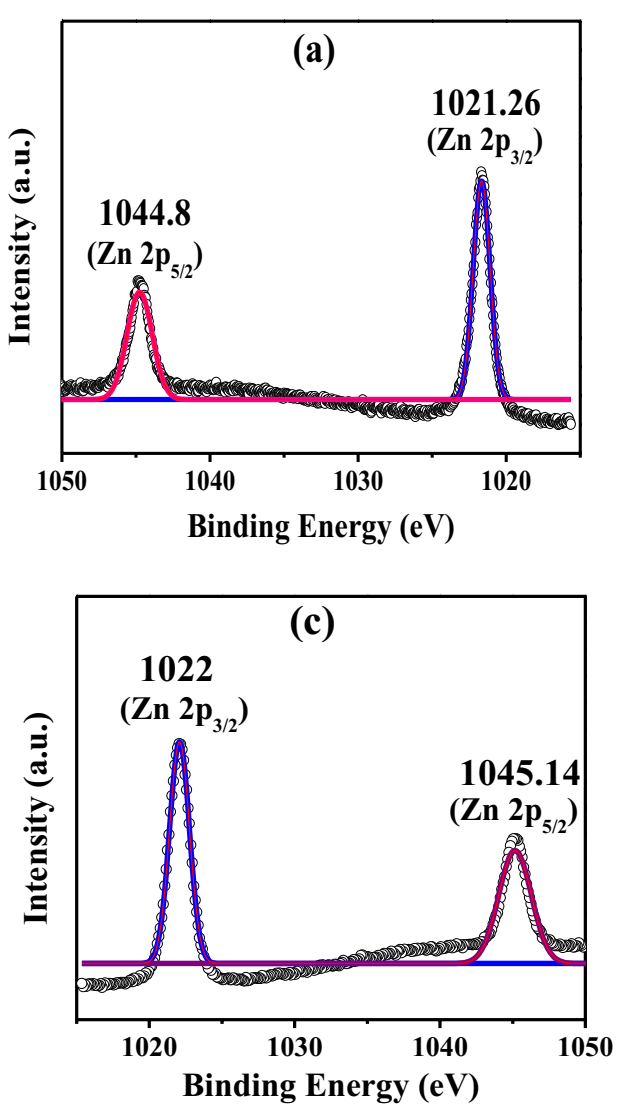
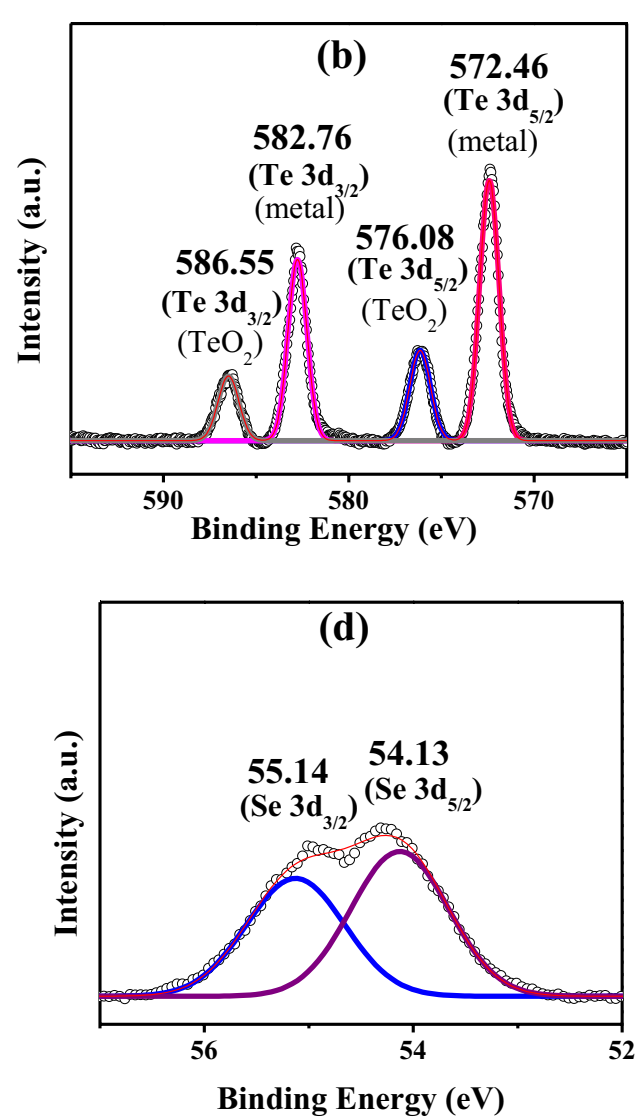

SN Applied Sciences a SPRINGER Nature journa 
the other two peaks at 586.55 and $576.08 \mathrm{eV}$ (though with less intensity) are attributed to the 2- oxidation state of Te from its oxide which might also be formed in very minute concentrations during the chemical synthesis. However, no such evidence has significantly been observed from other characteristic analytical techniques. In addition to that, Fig. $3 \mathrm{c}$ for $\mathrm{Zn}$ in ZnSe shows the similar phenomenon as already explained for $\mathrm{Zn}$ in ZnTe. Nevertheless, Se (Fig. 3d) shows two peaks for the $3 d$ scan at 55.14 and $54.13 \mathrm{eV}$ for its 2 - oxidation state.

\subsection{Alignment of energy levels}

The alignment of energy levels is an important parameter to understand the mechanism of photocatalytic reactions and the bandgap energies of constituent semiconductors as well as their valence band positions against the standard hydrogen electrodes (SHE) are two fundamental elements to further draw the alignment.

For this, the bandgap energies for both photocatalysts i.e. ZnTe and ZnSe, have been calculated from the UV-visible spectroscopy and the results are given in Fig. 4.
It can be seen in Fig. 4a that ZnTe shows a significance light absorption at around $559 \mathrm{~nm}$ corresponding to the bandgap energy of $2.22 \mathrm{eV}$ also given in the inset of Fig. $4 \mathrm{a}$ while using the Eq. 11. Furthermore, Fig. $4 \mathrm{~b}$ shows strong absorption of visible-light for $\mathrm{ZnSe}$ at $480 \mathrm{~nm}$ with the bandgap of $2.62 \mathrm{eV}$ as displayed in the inset.

On the other hand, the valence band edges for both ZnTe and ZnSe against SHE were determined via the XPS and the results are shown in Fig. 5. The zoom-in of the valence band edge spectrum is given in the respective insets to easily find the valence band position which are found to be $0.51 \mathrm{eV}(0.53 \mathrm{~V}$ vs. SHE) and $1.24 \mathrm{eV}(1.26 \mathrm{~V}$ vs. SHE) for ZnTe and ZnSe, respectively as shown in Fig. $5 a$, b. Based on Figs. 4, 5, the alignment of energy levels is plotted and is shown in the Fig. 6.

\subsection{Photocatalytic degradation of Congo red dye}

To check the photocatalytic activities, the as-synthesized samples including ZT, ZTS 1, ZTS 2, ZTS 3 and ZS were irradiated with UV-Vis light source to photodegrade the CR. For ZnTe, when it is irradiated with the light of energy
Fig. 4 UV-visible light absorption spectrum of $\mathbf{a} \mathrm{ZnTe}, \mathbf{b}$ ZnSe with insets showing the respective Tauc plots
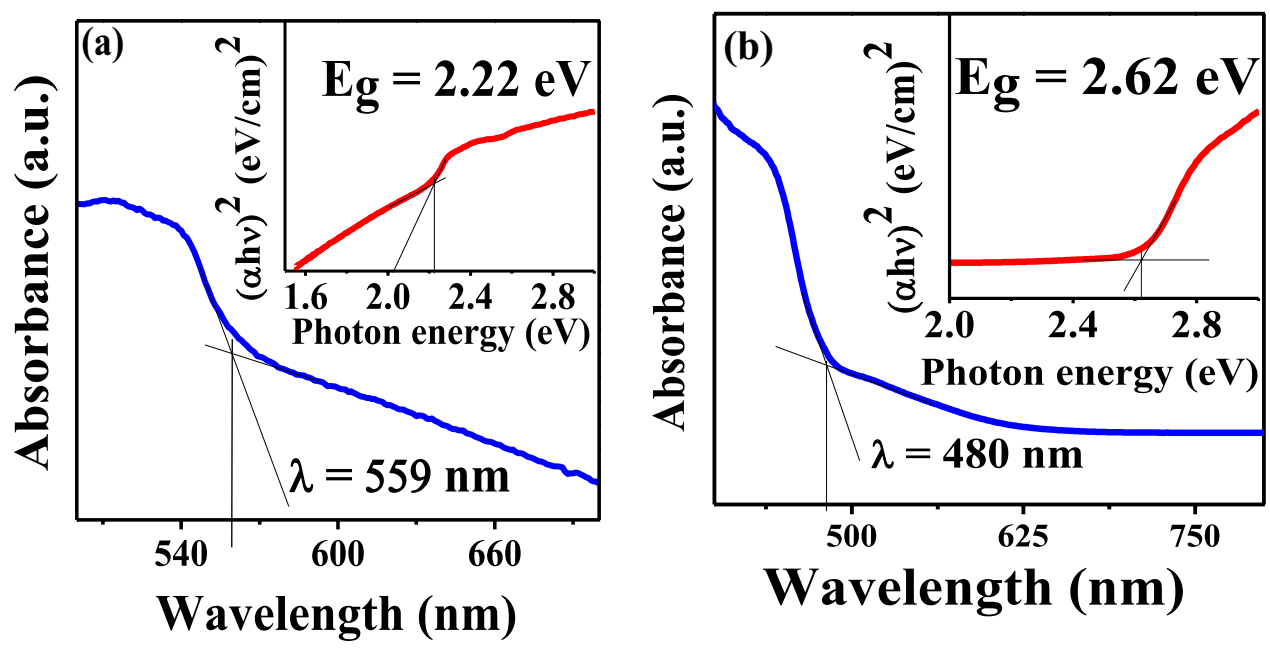

Fig. 5 XPS spectra of a ZnTe and $\mathbf{b} \mathrm{ZnSe}$ with the zoom-in images for the valence band positions in the respective insets
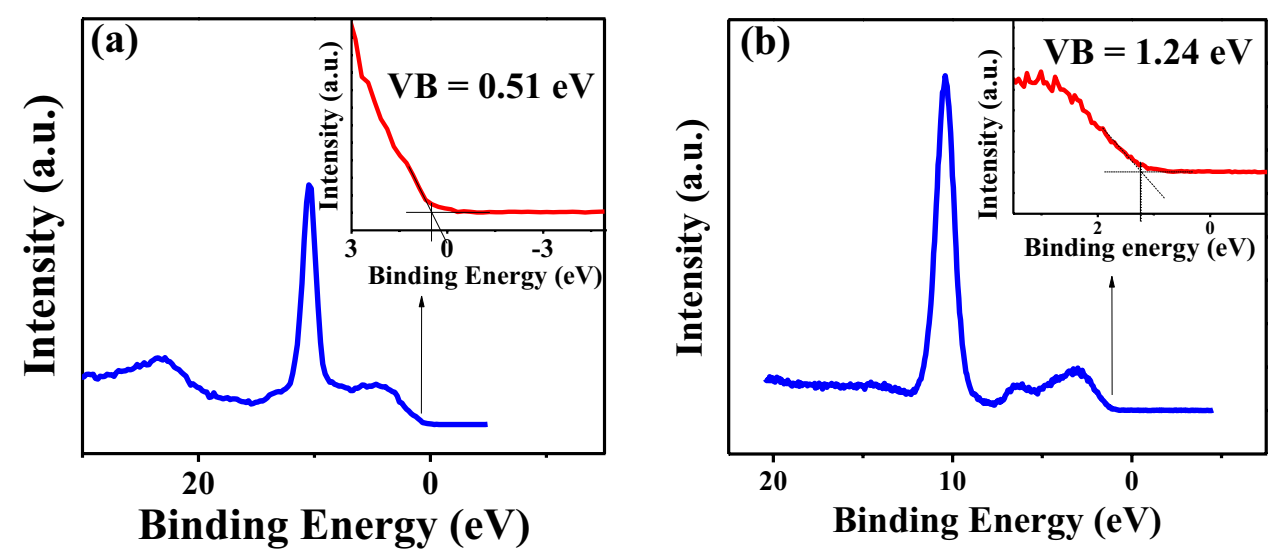

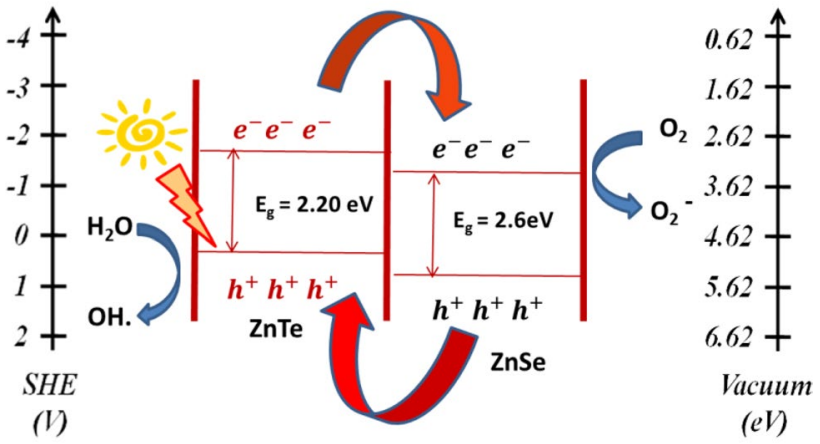

Fig. 6 Schematic illustration of alignment of energy levels of nanocomposites of ZnTe/ZnSe

either equal or greater than its bandgap energy, the electrons from its valence band (VB), while leaving the holes behind, are excited into the conduction band $(C B)$ and thus the redox reactions take place to mineralize the $C R$ dye. Similar phenomenon is carried out for the bare $\mathrm{ZnSe}$ photocatalysts. However, in case of the heterostructures as also shown in Fig. 6, the electrons from the CB of ZnTe are transferred to the $\mathrm{CB}$ of $\mathrm{ZnSe}$ owing to the thermodynamic feasibility while the holes from the VB of ZnSe are transferred into the VB of ZnTe thereby separating these charge carriers to avoid their recombination to hamper the overall photocatalytic activity. The electrons in the $\mathrm{CB}$ of $\mathrm{ZnSe}$ are responsible for the reduction of aqueous suspension thereby generating the superoxide anions while the holes in the VB of ZnTe oxidize the water molecules to generate hydroxyl radicals which together with the superoxide anions degrade the $C R$ dye. As shown in Fig. 7, when ZnTe was used as a photocatalysts, only $27.1 \%$ of dye molecules were degraded after $65 \mathrm{~min}$ under UV-Vis light irradiation. This lower degradation efficiency of ZnTe could be due to its rapid e-/h+ pair recombination which dissipates the solar energy in the form of heat. On the other hand, when ZnSe was used as a photocatalyst, about $64.1 \%$ of dye was degraded. The degradation efficiency of $\mathrm{ZnSe}$ was ascribed to its more suitable band positions as compared to ZnTe. In case of composite, ZTS 1, when the molar ratio of Se:Te was $1: 3,28.7 \%$ of the $C R$ molecules were degraded after $65 \mathrm{~min}$. With the increase in the amount of Se in the composite ZTS 2, the percentage degradation of Congo red increases to $94 \%$ after 65 min as shown in Fig. 7. The photocatalytic activity increases because of strong interface formation, while in case of ZTS 3 only $78.4 \%$ of dye was degraded. These results indicate that ZTS 2 shows the best photocatalytic activity which might be attributed to the reduced charge recombination. Furthermore, it was also noted that the presence of a common cation i.e.

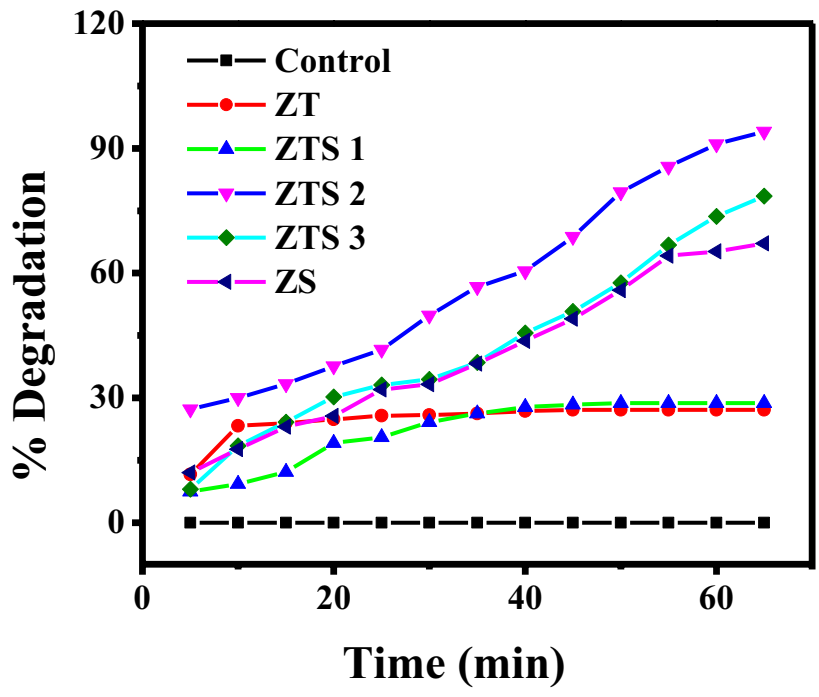

Fig. 7 Photocatalytic dye degradation for Congo red dye: a ZnTe, b-d composites, e ZnSe

$\mathrm{Zn}$, in the $\mathrm{ZnTe} / \mathrm{ZnSe}$ heterostructures might also play a significant role in enhancing the photodegradation of dye $[38,40]$. It might act as an additional barrier to hinder the charge recombination which is also responsible for the higher activities.

\section{Conclusion}

Hydrothermal approach has been employed as an efficient method for the in situ synthesis of heterostructures. The crystal structure, morphology and chemical composition of the as-synthesized ZnTe/ZnSe nanomaterials analyzed by various characterization techniques including the XRD, SEM and EDX/XPS, respectively confirm the impurity free synthesis of the heterostructured materials. In addition, the alignment of energy levels was successfully plotted based on the bandgap/valence band calculations which conclude the formation of type II heterostructure. The photocatalytic results reveal that both (1) the heterostructure formation and (2) concentration of constituents within the heterostructure are the key factors that facilitate the efficient charge separation and result in higher photocatalytic activities.

Acknowledgements M. F. Ehsan is thankful to the Higher Education Commission (HEC) of Pakistan for providing the financial Grant (No.: 6037/Federal/NRPU/R\&D/HEC/2016) under NRPU. The author also pays special thanks to Prof. Tao He from the National Center for Nanoscience and Technology Beijing and the Chinese Academy of 
Sciences for partial financial support from the Belt and Road initiative for the sample characterizations to carry out XPS analysis.

\section{Compliance with ethical standards}

Conflict of interest The authors declare that they have no conflict of interest.

\section{References}

1. Banat IM, Nigam P, Singh D et al (1996) Microbial decolorization of textile-dyecontaining effluents: a review. Bioresour Technol 58:217-227

2. Vandevivere PC, Bianchi R, Verstraete W et al (1998) Treatment and reuse of wastewater from the textile wet-processing industry: review of emerging technologies. J Chem Technol Biotechnol 72:289-302

3. Razo-Flores E, Luijten M, Donlon B et al (1997) Biodegradation of selected azo dyes under methanogenic conditions. Water Sci Technol 36:65-72

4. Sauer T, Neto GC, Jose HJ et al (2002) Kinetics of photocatalytic degradation of reactive dyes in a $\mathrm{TiO}_{2}$ slurry reactor. J Photochem Photobiol A Chem 149:147-154

5. Elahmadi MF, Bensalah N, Gadri A et al (2009) Treatment of aqueous wastes contaminated with Congo red dye by electrochemical oxidation and ozonation processes. J Hazard Mater 168:1163-1169

6. Kim J, Satoca DM, Choi W (2012) Simultaneous production of hydrogen with the degradation of organic pollutants using $\mathrm{TiO}_{2}$ photocatalyst modified with dual surface components. Energy Environ Sci 5:7647-7656

7. Fujishima A, Honda K (1972) Electrochemical photolysis of water at a semiconductor electrode. Nature 238:37-38

8. Lachheb H, Puzenat E, Houas A et al (2002) Photocatalytic degradation of various types of dyes (Alizarin $\mathrm{S}$, Crocein orange $\mathrm{G}$, Methyl red, Congo red, Methylene blue) in water by UV-irradiated titania. Appl Catal B Environ 39:75-90

9. Khan MM, Adil SF, Al-Mayouf A (2015) Metal oxides as photocatalysts. J Saudi Chem Soc 19:462-464

10. Schlesinger $M$, Weber $M$, Schulze $S$ et al (2013) Metastable $\beta-\mathrm{Bi}_{2} \mathrm{O}_{3}$ nanoparticles with potential for photocatalytic water purification using visible light irradiation. ChemistryOpen 2:146-155

11. Chen $D, Y e J(2008)$ Hierarchical $W_{3}$ hollow shells: dendrite, sphere, dumbbell, and their photocatalytic properties. Adv Funct Mater 18:1922-1928

12. Zhang L, Wang W, Chen $Z$ et al (2007) Fabrication of flower-like $\mathrm{Bi}_{2} \mathrm{WO}_{6}$ superstructures as high performance visible-light driven photocatalysts. J Mater Chem 17:2526-2532

13. Wang $\mathrm{H}$, Zhang L, Hu J et al (2014) Semiconductor heterojunction photocatalysts: design, construction, and photocatalytic performances. Chem Soc Rev 43:5234-5244

14. Ong WJ, Tan LL, Ng YH et al (2016) Graphitic carbon nitride $\left(\mathrm{g}-\mathrm{C}_{3} \mathrm{~N}_{4}\right.$ )-based photocatalysts for artificial photosynthesis and environmental remediation: Are we a step closer to achieving sustainability? Chem Rev 116:7159-7329

15. Saravanan R, Karthikeyan S, Gupta VK et al (2013) Enhanced photocatalytic activity of $\mathrm{ZnO} / \mathrm{CuO}$ nanocomposite for the degradation of textile dye on visible light illumination. Mater Sci Eng C Mater Biol Appl 33:91-98
16. Cun W, Jincai Z, Xinming W et al (2002) Preparation, characterization and photocatalytic activity of nano-sized $\mathrm{ZnO} / \mathrm{SnO}_{2} \mathrm{cou}-$ pled photocatalysts. Appl Catal B 39(3):269-279

17. Gopidas KR, Bohorquez M, Kamat PV (1990) Photophysical and photochemical aspects of coupled semiconductors: charge-transfer processes in colloidal cadmium sulfide-titania and cadmium sulfide-silver(I) iodide systems. J Phys Chem 94(16):6435-6440

18. Long M, Cai W, Cai J et al (2006) Efficient photocatalytic degradation of phenol over $\mathrm{Co}_{3} \mathrm{O}_{4} / \mathrm{BiVO}_{4}$ composite under visible light irradiation. J Phys Chem B 110(41):20211-20216

19. Xue $M$, Huang $L$, Wang JQ et al (2008) The direct synthesis of mesoporous structured $\mathrm{MnO}(2) / \mathrm{TiO}(2)$ nanocomposite: a novel visible-light active photocatalyst with large pore size. Nanotechnology 19(18):185604

20. Zhao L, Chen X, Wang X et al (2010) One-step solvothermal synthesis of a carbon@TiO(2) dyade structure effectively promoting visible-light photocatalysis. Adv Mater 22(30):3317-3321

21. Zhang $\mathrm{L}$, Wong $\mathrm{KH}$, Chen $\mathrm{Z}$ et al (2009) $\mathrm{AgBr}-\mathrm{Ag}-\mathrm{Bi}_{2} \mathrm{WO}_{6}$ nanojunction system: a novel and efficient photocatalyst with double visible-light active components. Appl Catal A Gen 363(1-2):221-229

22. Zhang LS, Wong KH, Yip HY et al (2010) Effective photocatalytic disinfection of $E$. coli $\mathrm{K}-12$ using $\mathrm{AgBr}-\mathrm{Ag}-\mathrm{Bi}_{2} \mathrm{WO}_{6}$ nanojunction system irradiated by visible light: the role of diffusing hydroxyl radicals. Environ Sci Technol 44(4):1392-1398

23. Wang $\mathrm{H}$, Li S, Zhang $\mathrm{L}$ et al (2013) Surface decoration of $\mathrm{Bi}_{2} \mathrm{WO}_{6}$ superstructures with $\mathrm{Bi}_{2} \mathrm{O}_{3}$ nanoparticles: an efficient method to improve visible-light-driven photocatalytic activity. CrystEngComm 15:9011-9019

24. Li G, Dimitrijevic NM, Chen L et al (2008) Role of surface/interfacial $\mathrm{Cu}^{2+}$ sites in the photocatalytic activity of coupled CuO$\mathrm{TiO}_{2}$ nanocomposites. J Phys Chem C 112(48):19040-19044

25. He Y, Wu Z, Fu L et al (2003) Photochromism and size effect of $\mathrm{WO}_{3}$ and $\mathrm{WO}_{3}-\mathrm{TiO}_{2}$ aqueous sol. Chem Mater 15(21):4039-4045

26. Marci G, Augugliaro V, Lopez-Munoz MJ et al (2001) Preparation characterization and photocatalytic activity of polycrystalline $\mathrm{ZnO} / \mathrm{TiO}_{2}$ systems. 1. Surface and bulk characterization. J Phys Chem B 105(5):1026-1032

27. Bandara J, Kuruppu SS, Pradeep UW (2006) The promoting effect of $\mathrm{MgO}$ layer in sensitized photodegradation of colorants on $\mathrm{TiO}_{2} / \mathrm{MgO}$ composite oxide. Colloids Surf A Physiochem Eng Asp 276:197-202

28. Cusano DA (1963) CdTe solar cells and photovoltaic heterojunctions in II-VI compounds. Solid State Electron 6(3):217-232

29. Afzaal M, O'Brian P (2006) Recent developments in II-VI and III$\mathrm{VI}$ semiconductors and their applications in solar cells. J Mater Chem 16(17):1597-1602

30. Anusorn K, Kevin T, Kensuke T et al (2008) Quantum dot solar cells. Tuning photoresponse through size and shape control of $\mathrm{CdSe}-\mathrm{TiO}_{2}$ architecture. J Phys Chem C 130:4007-4015

31. Kagkoura A, Skaltsas T, Tagmatarchis N (2017) Transition-metal chalcogenide/graphene ensembles for light-induced energy applications. Chem Eur J 23(53):12967-12979

32. Zhang L, Yang H, Yu J et al (2009) Controlled synthesis and photocatalytic activity of $\mathrm{ZnSe}$ nanostructured assemblies with different morphologies and crystalline phases. J Phys Chem C 113(14):5434-5443

33. Schrier J, Demchenko DO, Wang LW (2007) Optical properties of $\mathrm{ZnO} / \mathrm{ZnS}$ and $\mathrm{ZnO} / \mathrm{ZnTe}$ heterostructures for photovoltaic applications. Nano Lett 7(8):2377-2382

34. Tanaka T, Saito K, Guo Q et al (2009) Enhanced light output from ZnTe light emitting diodes by utilizing thin film structure. Appl Phys Exp 2(12):122101-122103 
35. Wu Q, Litz M, Zhang XC (1996) Broadband detection capability of ZnTe electro-optic field detectors. Appl Phys Lett 68:2924

36. Ehsan MF, Ashiq MN, Bi F et al (2014) Preparation and characterization of $\mathrm{SrTiO}_{3}-\mathrm{ZnTe}$ nanocomposites for visible-light photoconversion of carbon dioxide to methane. RSC Adv 4:48411-48418

37. Ehsan MF, Ashiq MN, He T (2015) Hollow and mesoporous ZnTe microspheres: synthesis and visible-light photocatalytic reduction of carbon dioxide into methane. RSC Adv 5:6186-6194

38. Ehsan MF, He T (2015) In-situ synthesis of ZnO/ZnTe common cation heterostructure and its visible-light photocatalytic reduction of $\mathrm{CO}_{2}$ into $\mathrm{CH}_{4}$. Appl Catal B Environ 166-167:345-352
39. Ehsan MF, Khan R, He T (2017) Visible-light photoreduction of $\mathrm{CO}_{2}$ into $\mathrm{CH}_{4}$ over $\mathrm{ZnTe}$-modified $\mathrm{TiO}_{2}$ coral-like nanostructures. ChemPhysChem 18:3203-3210

40. Zia A, Hamid S, Ehsan MF et al (2018) Congo red photomineralization over $\mathrm{Co}_{3} \mathrm{O}_{4} / \mathrm{CoTe}$ common cation nanocomposites. J Mater Sci Mater Electron 29:20271-20279

Publisher's Note Springer Nature remains neutral with regard to jurisdictional claims in published maps and institutional affiliations. 